\title{
The Value of CT Imaging and Psoas Muscle Index in Grading the Severity of Sarcopenia in Liver Cirrhosis Patients and its Impact on Morbidity and Mortality
}

\author{
HEBA KAMAL, M.D. and NEVIEN EL-LEITHY, M.D. \\ The Department of Diagnostic and Interventional Radiology, Faculty of Medicine, Cairo University
}

\begin{abstract}
Background: Sarcopenia is a common feature of malnutrition in patient with liver cirrhosis and is widely recognized as independent predictor of poor outcome and mortality in this setting.
\end{abstract}

Aim of Study: This work was conducted to assess the severity of sarcopenia in liver cirrhosis patients, by quantifying muscle mass through CT at L3 level, measuring psoas muscle volume and cross sectional area, than correlating the results with hand grip strength as representative of functional status.

Patients and Methods: This study included 101 liver cirrhosis patients and 30 controls. All enrolled subjects had abdominal computed tomographic (CT) imaging at the level of L3 to calculate Psoas muscle cross sectional area, volume, and psoas muscle index (PMI) which is the sum of both RT and LT cross sectional area/height ${ }^{2}\left(\mathrm{~m}^{2}\right)$, also hand grip strength was calculated for all patients using hand grip dynamometry. The severity of cirrhosis was classified according to the Child-Pugh and MELD scores.

Result: This was an observational prospective study including (101 liver cirrhosis \& 30 controls), the mean age was $59.67 \mathrm{ys} \pm 8.01$, cirrhotic patients were graded into three groups according to Child scoring system. Mean CT psoas volume, psoas surface area and psoas muscle index for Child $(\mathrm{A}+\mathrm{B})$ were $25.44 \pm 6.96,7.97 \pm 2.23 \& 4.58 \pm 1.21$, while for Child C, were $15.85 \pm 6.02,5.02 \pm 1.80 \& 2.96 \pm 1.00$. Using the FNIH (Foundation for the national Institutes of health) cuff off of hand grip $(<26 \mathrm{~kg}$ in male and $<16 \mathrm{~kg}$ in female Psoas muscle index cut off was $4.5 \mathrm{~cm}^{2} / \mathrm{m}^{2}$

Conclusion: Psoas muscle cross sectional area, volume and index measurements were well correlated with hand grip strength and proved to be independent prognostic factor for grading sarcopenia in cirrhotic patients.

Key Words: Sarcopenia-CT-Psoas muscle-Handgrip strength.

Correspondence to: Dr. Heba Kamal, The Department of Diagnostic and Interventional Radiology, Kasr Al-Aini, Faculty of Medicine, Cairo University

\section{Introduction}

SARCOPENIA is defined as a regression in the muscle mass, muscle strength and physical activity [1]. It is associated with defective physical capability, poor quality of life, detrimental metabolic effects, disability, mortality, and high health care expenses [2].

Malnutrition and sarcopenia are highly common in patients with advanced and chronic liver disease, and they are frequently common in patients with liver cirrhosis [3]. Once sarcopenia develops, prognosis is markedly declined and patients are at high risk for liver related morbidity and mortality [4]

Conventional prognostic scores for patients with cirrhosis, such as the Child-Turcotte-Pugh (CTP) score or the model for end-stage liver diseases (MELD) score, have limitations, including the lack of a nutritional status evaluation [5]. This may be caused by the lack of a clear definition and the complexity of a nutritional assessment in patients with cirrhosis and fluid overload [6]. Some

\begin{tabular}{|c|c|}
\hline \multicolumn{2}{|c|}{ List of Abbreviations: } \\
\hline BMI & : Body mass index \\
\hline FNIH & : Foundation for National Institutes of Health \\
\hline HGS & : Hand grip strength \\
\hline HU & : Hounsfield units \\
\hline MUAC & : Mid upper arm circumference \\
\hline SD & : Standard deviation \\
\hline CT & : Computed tomography \\
\hline LT & : Left \\
\hline RT & : Right \\
\hline PMI & : Psoas muscle index \\
\hline SMI & : Skeletal muscle index \\
\hline MELD & : Model of end stage liver disease \\
\hline СТP & : Child-Turcotte-Pugh \\
\hline SMA & : Skeletal muscle area \\
\hline SPSS & : SPSS statistical package for the Social Sciences \\
\hline PACS & archiving and con \\
\hline
\end{tabular}


of these reports suggest that sarcopenia adds to the prognostic value of the model for end-stage liver disease (MELD) scoring system [7].

Different imaging modalities is being progressively used to diagnose and estimate the degree of sarcopenia in patients with cirrhosis, including DEXA, CT, MRI and ultrasonography [2]. Computed tomography (CT) represents the gold standard for diagnosing sarcopenia, [8] since it has the capacity to differentiate between different body tissues (skeletal muscle, adipose tissue, bone, water and air) based on tissue specific attenuation values, calculated in Hounsfield units (HU) [10]

CT scanning through specific software allows measuring of skeletal muscle area (SMA), which when adjusted for height, generates the skeletal muscle index (SMI), which is being the most widely used parameter. SMI is generated from the cross sectional area of abdominal skeletal muscles at the third lumbar vertebrae, normalized by body height $[8,11]$.

SMI is known to be an independent prognostic factor of mortality in cirrhotic patients [2] . Abdominal muscles, including the psoas muscle, are appropriate to use for the estimation of skeletal muscle mass, because abdominal muscle mass, unlike appendicular muscle mass, is generally independent of activity level [2] .

There is increasing evidence showing that Voluntary handgrip strength (HGS) is an indicator of nutrition status [12] and a promising undernutrition screening tool. It is an indicator of overall body muscle strength [13] and can delineate effects of nutrition deprivation, before alterations in body composition parameters are identifiable [14]

The Foundation for the National Institutes of Health Sarcopenia Project [15] recommends specific cutoff points to identify populations with functional limitations associated with sarcopenia. These evidence-based criteria recognize measures of low muscle strength (i.e., grip strength $<26 \mathrm{~kg}$ for men and $<16 \mathrm{~kg}$ for women) and low lean body mass (i.e., appendicular lean mass adjusted for body mass index (BMI; weight in kilograms divided by the square of height in meters) $<0.789$ for men and $<0.512$ for women) [16]. These criteria were validated as predictive of future mobility impairment with a 3-year clinical follow-up, 16 and this definition of muscle weakness appears to be a treatable symptom of sarcopenia [17].

In the present study, we primarily aimed to verify the concordance between muscle wasting, determined by CT scan with other techniques providing muscle functions such as the HG test. As a secondary aim, we evaluated the correlation between muscle wasting, degree of liver cirrhosis, impairment, and survival.

\section{Patients and Methods}

\section{Patients:}

The current study was an observational prospective study conducted during the period from January 2020 to January 2021, including 101 cirrhotic patients admitted to the Internal Medicine Hospital, Kaser Al-Eini, Cairo University, being referred either from the outpatient clinics or from the emergency department, also 30 control participants were included within the study, to validate the role of CT measures in assessing the severity of sarcopenia in liver cirrhosis.

The ethics commission of our institution approved this study and written informed consent for the current study from all patients before enrollment was obtained. Patients were fully informed about the risks and benefits of the radiological procedures; also they were informed about the research plan.

\section{Inclusion criteria:}

The criteria for enrollment were as follows:

- Adult patients previously diagnosed with liver cirrhosis either by biopsy or abdominal ultrasound (based on the morphologic characteristics of cirrhosis, including hepatic contour, texture, and existing portal collaterals).

- Patients admitted to our hospital by a complication of liver cirrhosis (hematemesis, spontaneous bacterial peritonitis, hepatic encephalopathy, hepatorenal syndrome).

\section{Exclusion criteria for:}

1- History of end organ failure which interfere with nutritional status e.g end stage renal failure on hemodialysis, heart failure, and respiratory failure).

2- Malignancies and hepatocellular carcinoma.

3- Acute liver cell failure.

\section{All patients were submitted to the following:}

1 - History taking.

2- Full physical examination, (including BMI, nutritional risk score (NRS), anthropometric measurements), additionally to the clinical features and extent of the underlying pathology. The severity of cirrhosis was classified according to the Child-Pugh and MELD score. 
3- Laboratory investigations including liver function tests and kidney functions.

Image analysis:

CT protocol:

- CT was performed using (Siemens Healthcare emotion 16 multidetector CT scan, Erlangen Germany).

- CT was done as a part of the overall investigations done for the cirrhotic patients.

- Single axial CT image at the level of L3 was utilized to outline both psoas muscles, using tube voltage of $130 \mathrm{kV}$, tube current $50-200 \mathrm{~mA}$, exposure time: $100-300 \mathrm{mAs}$, slice thickness $10 \mathrm{~mm}$, tissue-specific Hounsfield unit thresholds of -30 to +200 to separate muscle tissue from fat tissue or bone.

- Images were analyzed through picture archiving and communication system (PACS). Within the generated CT image, the borders of the right and left psoas muscles were manually drawn within the axial sections passing through the mid part of L3 vertebra.

- Volume of psoas muscle $\left(\mathrm{cm}^{3}\right)$ (Fig. 1) and crosssectional area $\left(\mathrm{cm}^{2}\right)$ at L3 level (Fig. 2), were calculated automatically by adding tissue pixels and multiplying by the pixel surface area using Ultima program.

- The total psoas area was calculated by the sum of left and right psoas areas, then normalized by using the patient's height to produce psoas muscle index (PMI) in $\mathrm{cm}^{2} / \mathrm{m}^{2}$.

- Height correction is crucial to assess the relative muscle mass due to the linear relationship between skeletal muscle and height.

- Psoas muscle index (PMI) in $\mathrm{cm}^{2} / \mathrm{m}^{2}$ at L3 = Total psoas muscle area at $\mathrm{L} 3$ level $\mathrm{cm}^{2} /$ height $\left(\mathrm{m}^{2}\right)$

- The attenuation of muscle was also measured to detect muscle quality. Low muscle attenuation, described as myosteatosis, which is defined by increased intramuscular fat content, which contributes to muscle weakness irrespective of the age-associated loss in muscle mass.

\section{Assessment of muscle function:}

Hand Grip strength (HGS):

It was measured using the hand grip dynamometry shown in (Fig. 3). The Patient's data in the form of age and sex were recorded then the patient was asked to use their maximum effort to grip both handles of the dynamometer together. The test was repeated for 3 times for every hand separately then the highest number value was used as a predictor of patient's performance. According to the Foundation for national institutes of health sarcopenia project, which identified a cut off for muscle strength as $<26 \mathrm{~kg}$ for men and $<16 \mathrm{~kg}$ for women.

\section{Correlation of CT measurements and HGS:}

Comparative analysis was done between the CT findings with the muscle function using the Hand Grip strength (HGS), then the results were correlated with the normal cut off values for age and sex, as outlined within the results.

\section{Statistical methods:}

Data were coded and entered using the statistical package for the Social Sciences (SPSS) version 25 (IBM Corp., Armonk, NY, USA). Data was summarized using mean and standard deviation for quantitative variables, frequencies (number of cases) and relative frequencies (percentages) for categorical variables. Comparisons between groups were done using unpaired $t$-test, when comparing 2 groups and analysis of variance (ANOVA) with multiple comparisons post hoc test when comparing more than 2 groups. For comparing categorical data, Chi square test was performed. Exact test was used instead when the expected frequency is less than 5 .

Correlations between quantitative variables were done using Pearson correlation coefficient. $p$-values less than 0.05 were considered as statistically significant.

\section{Results}

The present study was conducted on 101 liver cirrhosis patients, who were admitted in internal medicine hospital. In addition to 30 health participants as control group Tables $(1,2)$.

According to Child score, patients were divided into 3 groups: Child A (6 patients), Child B (20 patients) and Child C (74 patients). The data of Child A and Child B patients were analyzed as one group in comparison to child c patients.

Control group constituted of 30 healthy participants with mean age 59.67ys \pm 8.01 .

\section{Hand grip and anthropometric measures in Child groups:}

The hand grip and anthropometric measures in Child groups namely dry weight, BMI, Mid upper arm circumference (MUAC) differed significantly between patients and control as well as between both groups of patients ( $p$-value $<0.001)$. Table (3). 
Hand grip showed significant positive correlation with anthropometric measurements (BMI, MAUC), and significant negative correlation with disease severity score (Child score), mortality risk (MELD) and nutritional assessment. Table (4).

Table (1): Demographic data of the studied group.

\begin{tabular}{|c|c|c|c|}
\hline & $\begin{array}{c}\text { Child A,B } \\
\text { N (26) }\end{array}$ & $\begin{array}{l}\text { Child C } \\
\text { N (75) }\end{array}$ & $\begin{array}{l}\text { Control } \\
\mathrm{N}(30)\end{array}$ \\
\hline \multicolumn{4}{|l|}{ Age: } \\
\hline$<60$ & 8 & 46 & 14 \\
\hline$>60$ & 18 & 29 & 16 \\
\hline \multicolumn{4}{|l|}{ Sex: } \\
\hline Male/Female & $19 / 7$ & $36 / 39$ & $16 / 14$ \\
\hline \multicolumn{4}{|l|}{ Cause of admission: } \\
\hline Hematemesis & 24 & 48 & \\
\hline Hepatic coma & 2 & 21 & \\
\hline SBP & 0 & 5 & \\
\hline Hepto-renal & 0 & 1 & \\
\hline \multicolumn{4}{|l|}{ Etiology: } \\
\hline Hemochromatosis: & 0 & 1 & \\
\hline $\mathrm{HCV}$ & 21 & 65 & \\
\hline HBV & 0 & 5 & \\
\hline Bilharziasis & 5 & 3 & \\
\hline Autoimmune & 0 & 1 & \\
\hline \multicolumn{4}{|l|}{ Ascites: } \\
\hline Tense & 0 & 55 & \\
\hline Moderate & 20 & 20 & \\
\hline Non & 6 & 0 & \\
\hline \multicolumn{4}{|l|}{ ll edema: } \\
\hline Present & 20 & 74 & \\
\hline Non & 6 & 1 & \\
\hline
\end{tabular}

Table (2): Model for End-Stage Liver Disease (MELD), nutrition risk score (NRS) and length of hospital stay.

\begin{tabular}{lccc}
\hline & $\begin{array}{c}\text { Child A,B } \\
\text { Mean } \pm \text { SD }\end{array}$ & $\begin{array}{c}\text { Child C } \\
\text { Mean } \pm \text { SD }\end{array}$ & $\begin{array}{c}p \text { - } \\
\text { value }\end{array}$ \\
\hline MELD & $11.58 \pm 3.19$ & $22.41 \pm 6.71$ & $<0.001$ \\
NRS & $2.42 \pm 0.86$ & $4.05 \pm 0.77$ & $<0.001$ \\
Length of hospital & $3.38 \pm 0.80$ & $7.08 \pm 2.53$ & $<0.001$ \\
$\quad$ stay (days) & & & \\
\hline
\end{tabular}

Table (3): Hand grip and anthropometric measures.

\begin{tabular}{lcccc}
\hline & \multicolumn{3}{c}{ Group } & \\
\cline { 2 - 4 } & $\begin{array}{c}\text { Child A,B } \\
\text { Mean } \pm \text { SD }\end{array}$ & $\begin{array}{c}\text { Child C } \\
\text { Mean } \pm \text { SD }\end{array}$ & $\begin{array}{c}\text { Control } \\
\text { Mean } \pm \text { SD }\end{array}$ & \\
\hline value \\
Dry wt (kg) & $61.69 \pm 7.54$ & $51.73 \pm 5.22$ & $84.52 \pm 12.45$ & $<0.001$ \\
BMI & $20.59 \pm 1.69$ & $18.27 \pm 0.80$ & $28.21 \pm 4.89$ & $<0.001$ \\
MUAC & $28.31 \pm 3.16$ & $21.28 \pm 3.47$ & $33.45 \pm 2.52$ & $<0.001$ \\
Hand grip & $22.78 \pm 6.36$ & $14.88 \pm 5.13$ & $29.15 \pm 7.50$ & $<0.001$ \\
\hline
\end{tabular}

Table (4): Correlation between HGS, MAUC, BMI, MELD, Child score, NRS.

\begin{tabular}{lcccccc}
\hline & $\begin{array}{c}\text { Hand } \\
\text { grip }\end{array}$ & MELD & Child & NRS & MUAC & BMI \\
\hline $\begin{array}{l}\text { Hand grip: } \\
\text { Pearson }\end{array}$ & 1 & $-0.463-$ & $-0.542-$ & $-0.645-$ & 0.878 & 0.726 \\
$\begin{array}{l}\text { Correlation } \\
p \text {-value }\end{array}$ & & $<0.001$ & $<0.001$ & $<0.001$ & $<0.001$ & $<0.001$ \\
$\mathrm{~N}$ & 101 & 101 & 101 & 101 & 101 & 101 \\
\hline
\end{tabular}

\section{Muscle assessment by $C T$ :}

CT measurements of the psoas muscle at L3, including volume, surface area and psoas muscle index in Child groups were significantly decreased in patients when compared to control group Tables $(5,6)$, Figs. $(4,5)$.

Psoas CT at L3 CT measurements of the psoas muscle at L3, including Volume, surface area and Psoas muscle index (right, left), were significantly decreased with increasing disease severity, it was worst in Child $\mathrm{C}$ patients compared to Child A,B patients ( $p$-value <0.001) Table (7), Figs. $(6,7)$.

All CT parameters have significant positive correlation with HGS.

Also they have significant negative correlation with (Child, MELD, and NRS) scores and length of hospital stay with $(p$-value $<0.001)$ Table $(8)$.

Cut off values for male psoas index (Fig. 8):

It is illustrated in Table (8) with good sensitivity $84.1 \%$ and good specificity $100 \%$. Table (7).

Cut off values for female psoas index (Fig. 9):

It is illustrated in Table (9) with good sensitivity $93 \%$ and good specificity $100 \%$.

In general the CT L3 psoas volume, surface area, and psoas index on both sides with mean measure, showed significant positive correlation with anthropometric measurement, hand grip, and laboratory results (alb, Bil, INR, creat) ( $p$-value $<0.001)$. Table (10).

Table (5): Computerized tomography at L3 volume, surface area and psoas muscle index in Child groups.

\begin{tabular}{|c|c|c|c|c|}
\hline & \multicolumn{3}{|c|}{ Group } & \multirow{2}{*}{$\begin{array}{c}p- \\
\text { value }\end{array}$} \\
\hline & $\begin{array}{l}\text { Child A,B } \\
\text { Mean } \pm \text { SD }\end{array}$ & $\begin{array}{c}\text { Child C } \\
\text { Mean } \pm \text { SD }\end{array}$ & $\begin{array}{c}\text { Control } \\
\text { Mean } \pm \text { SD }\end{array}$ & \\
\hline $\begin{array}{l}\text { RT CT Psoas } \\
\text { volume }\end{array}$ & $25.12 \pm 6.87$ & $15.64 \pm 6.03$ & $30.06 \pm 9.85$ & $<0.001$ \\
\hline $\begin{array}{l}\text { LT CT Psoas } \\
\text { volume }\end{array}$ & $25.76 \pm 7.11$ & $16.06 \pm 6.09$ & $30.69 \pm 10.52$ & $<0.001$ \\
\hline $\begin{array}{l}\text { Mean RT \& LT CT } \\
\text { Psoas volume }\end{array}$ & $25.44 \pm 6.96$ & $15.85 \pm 6.02$ & $30.38 \pm 10.17$ & $<0.001$ \\
\hline $\begin{array}{l}\text { RT CT Psoas } \\
\text { surface area }\end{array}$ & $7.84 \pm 2.15$ & $4.99 \pm 1.78$ & $10.25 \pm 2.32$ & $<0.001$ \\
\hline $\begin{array}{l}\text { LT CT Psoas } \\
\text { surface area }\end{array}$ & $8.11 \pm 2.34$ & $5.06 \pm 1.89$ & $10.50 \pm 2.41$ & $<0.001$ \\
\hline $\begin{array}{l}\text { Mean RT \& LT CT } \\
\text { Psoas surface area }\end{array}$ & $7.97 \pm 2.23$ & $5.02 \pm 1.80$ & $10.37 \pm 2.34$ & $<0.001$ \\
\hline CT Psoas ms index & $4.58 \pm 1.21$ & $2.96 \pm 1.00$ & $5.94 \pm 1.24$ & $<0.001$ \\
\hline
\end{tabular}


Table (6): Post hoc pairwise comparison ( $p$-value between each 2 groups).

\begin{tabular}{lccc}
\hline & $\begin{array}{c}\text { Child A,B vs } \\
\text { Child C }\end{array}$ & $\begin{array}{c}\text { Child A,B vs } \\
\text { Control }\end{array}$ & $\begin{array}{c}\text { Child C vs } \\
\text { Control }\end{array}$ \\
\hline RT CT Psoas Volume & $<0.001$ & 0.036 & $<0.001$ \\
LT CT Psoas volume & $<0.001$ & 0.046 & $<0.001$ \\
Mean RT\&LT CT Psoas Volume & $<0.001$ & 0.040 & $<0.001$ \\
RT CT Psoas surface area & $<0.001$ & $<0.001$ & $<0.001$ \\
LT CT Psoas surface area & $<0.001$ & $<0.001$ & $<0.001$ \\
Mean RT\&LT CT Psoas surface area & $<0.001$ & $<0.001$ & $<0.001$ \\
CT Psoas ms index & $<0.001$ & $<0.001$ & $<0.001$ \\
\hline
\end{tabular}

Table (7): Correlation between CT parameters, (Child, MELD, and NRS) scores, hand grip, and length of hospital stay.

\begin{tabular}{|c|c|c|c|c|c|c|}
\hline & Child & MELD & NRS & MUAC & $\begin{array}{l}\text { Hand } \\
\text { grip }\end{array}$ & $\begin{array}{l}\text { Length of hospital } \\
\text { stay (days) }\end{array}$ \\
\hline \multicolumn{7}{|c|}{ RT CT Psoas Volume: } \\
\hline$r$ & $-0.570-$ & $-0.488-$ & $-0.674-$ & 0.898 & 0.964 & $-0.498-$ \\
\hline$p$-value & $<0.001$ & $<0.001$ & $<0.001$ & $<0.001$ & $<0.001$ & $<0.001$ \\
\hline $\mathrm{N}$ & 101 & 101 & 101 & 101 & 101 & 101 \\
\hline \multicolumn{7}{|c|}{ LT CT Psoas volume: } \\
\hline$r$ & $-0.582-$ & $-0.495-$ & $-0.678-$ & 0.899 & 0.971 & $-0.520-$ \\
\hline$p$-value & $<0.001$ & $<0.001$ & $<0.001$ & $<0.001$ & $<0.001$ & $<0.001$ \\
\hline $\mathrm{N}$ & 101 & 101 & 101 & 101 & 101 & 101 \\
\hline \multicolumn{7}{|c|}{ Mean RT\&LT CT Psoas Volume: } \\
\hline$r$ & $-0.578-$ & $-0.494-$ & $-0.679-$ & 0.902 & 0.971 & $-0.511-$ \\
\hline$p$-value & $<0.001$ & $<0.001$ & $<0.001$ & $<0.001$ & $<0.001$ & $<0.001$ \\
\hline $\mathrm{N}$ & 101 & 101 & 101 & 101 & 101 & 101 \\
\hline \multicolumn{7}{|c|}{ RT CT Psoas surface area: } \\
\hline$r$ & $-0.555-$ & $-0.478-$ & $-0.638-$ & 0.886 & 0.951 & $-0.481-$ \\
\hline$p$-value & $<0.001$ & $<0.001$ & $<0.001$ & $<0.001$ & $<0.001$ & $<0.001$ \\
\hline $\mathrm{N}$ & 101 & 101 & 101 & 101 & 101 & 101 \\
\hline \multicolumn{7}{|c|}{ LT CT Psoas surface area: } \\
\hline$r$ & $-0.570-$ & $-0.495-$ & $-0.644-$ & 0.883 & 0.960 & $-0.519-$ \\
\hline$p$-value & $<0.001$ & $<0.001$ & $<0.001$ & $<0.001$ & $<0.001$ & $<0.001$ \\
\hline $\mathrm{N}$ & 101 & 101 & 101 & 101 & 101 & 101 \\
\hline \multicolumn{7}{|c|}{ Mean RT\&LT CT Psoas surface area: } \\
\hline$r$ & $-0.568-$ & $-0.492-$ & $-0.648-$ & 0.893 & 0.965 & $-0.506-$ \\
\hline$p$-value & $<0.001$ & $<0.001$ & $<0.001$ & $<0.001$ & $<0.001$ & $<0.001$ \\
\hline $\mathrm{N}$ & 101 & 101 & 101 & 101 & 101 & 101 \\
\hline \multicolumn{7}{|c|}{ CT Psoas ms index: } \\
\hline$r$ & $-0.580-$ & $-0.516-$ & $-0.656-$ & 0.892 & 0.960 & $-0.521-$ \\
\hline$p$-value & $<0.001$ & $<0.001$ & $<0.001$ & $<0.001$ & $<0.001$ & $<0.001$ \\
\hline $\mathrm{N}$ & 101 & 101 & 101 & 101 & 101 & 101 \\
\hline
\end{tabular}

Table (8): Cut off values for male psoas index.

\begin{tabular}{|c|c|c|c|c|c|c|}
\hline \multirow{2}{*}{$\begin{array}{l}\text { Area } \\
\text { Under } \\
\text { the } \\
\text { Curve }\end{array}$} & \multirow{2}{*}{$\begin{array}{c}p- \\
\text { value }\end{array}$} & \multicolumn{2}{|c|}{$\begin{array}{l}\text { 95\% Confidence } \\
\text { Interval } \\
\end{array}$} & \multirow{2}{*}{$\begin{array}{l}\text { Cut off } \\
\left(\mathrm{cm}^{2} / \mathrm{m}^{2}\right)\end{array}$} & \multirow{2}{*}{$\begin{array}{c}\text { Sensitivity } \\
\%\end{array}$} & \multirow{2}{*}{$\begin{array}{c}\text { Specificity } \\
\%\end{array}$} \\
\hline & & $\begin{array}{l}\text { Lower } \\
\text { Bound }\end{array}$ & $\begin{array}{l}\text { Upper } \\
\text { Bound }\end{array}$ & & & \\
\hline 0.969 & $<0.001$ & 0.928 & 1.000 & 4.66 & 84.1 & 100 \\
\hline
\end{tabular}

Table (9): Cut off values for female psoas index.

\begin{tabular}{|c|c|c|c|c|c|c|}
\hline \multirow{2}{*}{$\begin{array}{l}\text { Area } \\
\text { Under } \\
\text { the } \\
\text { Curve }\end{array}$} & \multirow{2}{*}{$\begin{array}{c}p- \\
\text { value }\end{array}$} & \multicolumn{2}{|c|}{$\begin{array}{l}95 \% \text { Confidence } \\
\text { Interval }\end{array}$} & \multirow{2}{*}{$\begin{array}{l}\text { Cut off } \\
\left(\mathrm{cm}^{2} / \mathrm{m}^{2}\right)\end{array}$} & \multirow{2}{*}{$\begin{array}{c}\text { Sensitivity } \\
\%\end{array}$} & \multirow{2}{*}{$\begin{array}{c}\text { Specificity } \\
\%\end{array}$} \\
\hline & & $\begin{array}{l}\text { Lower } \\
\text { Bound }\end{array}$ & $\begin{array}{l}\text { Upper } \\
\text { Bound }\end{array}$ & & & \\
\hline 0.977 & 0.006 & 0.927 & 1.000 & 3.19 & 93 & 100 \\
\hline
\end{tabular}


Table (10): Correlation between CT L3 psoas volume, surface area, and psoas index with anthropometric measurement and laboratory results.

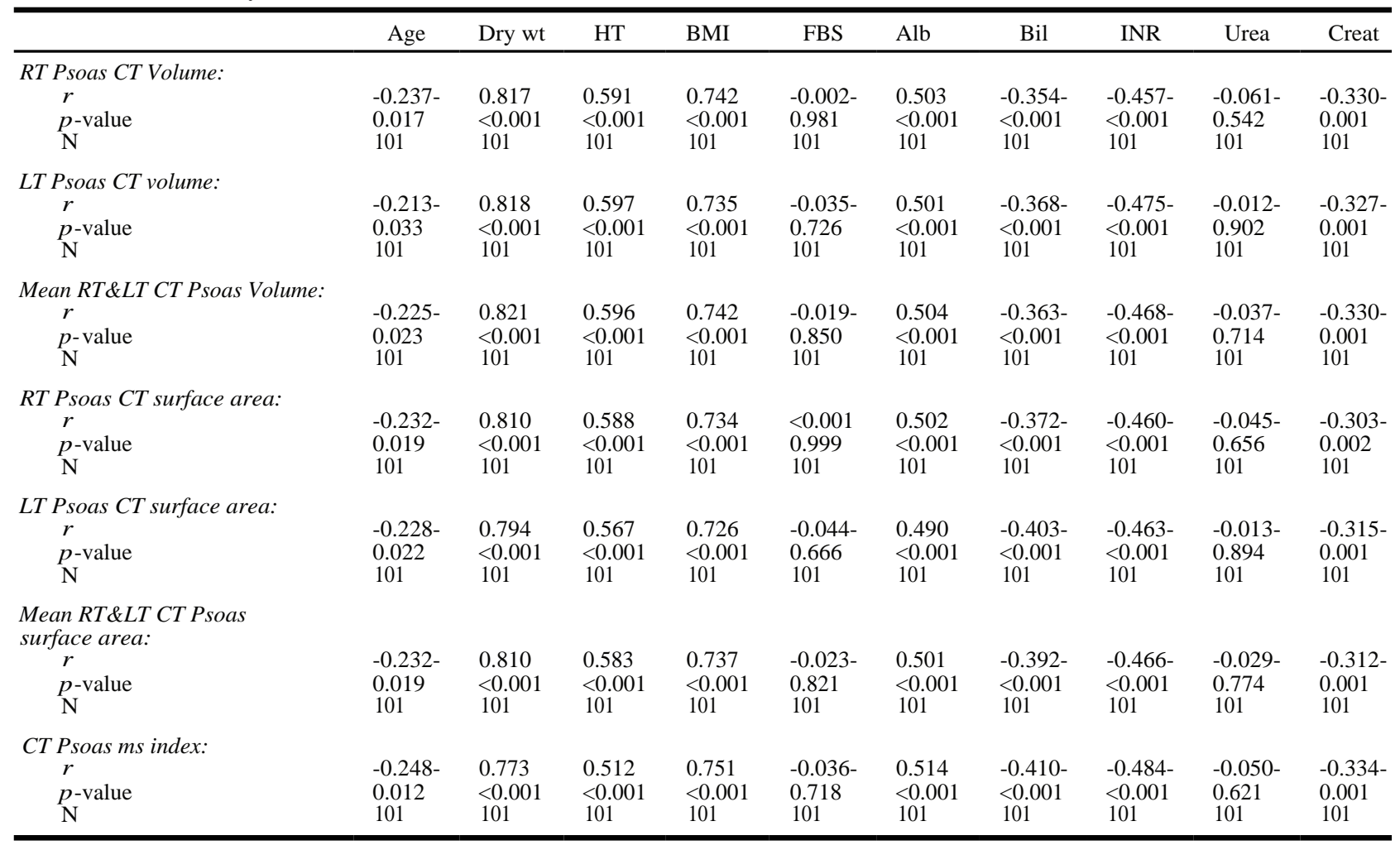
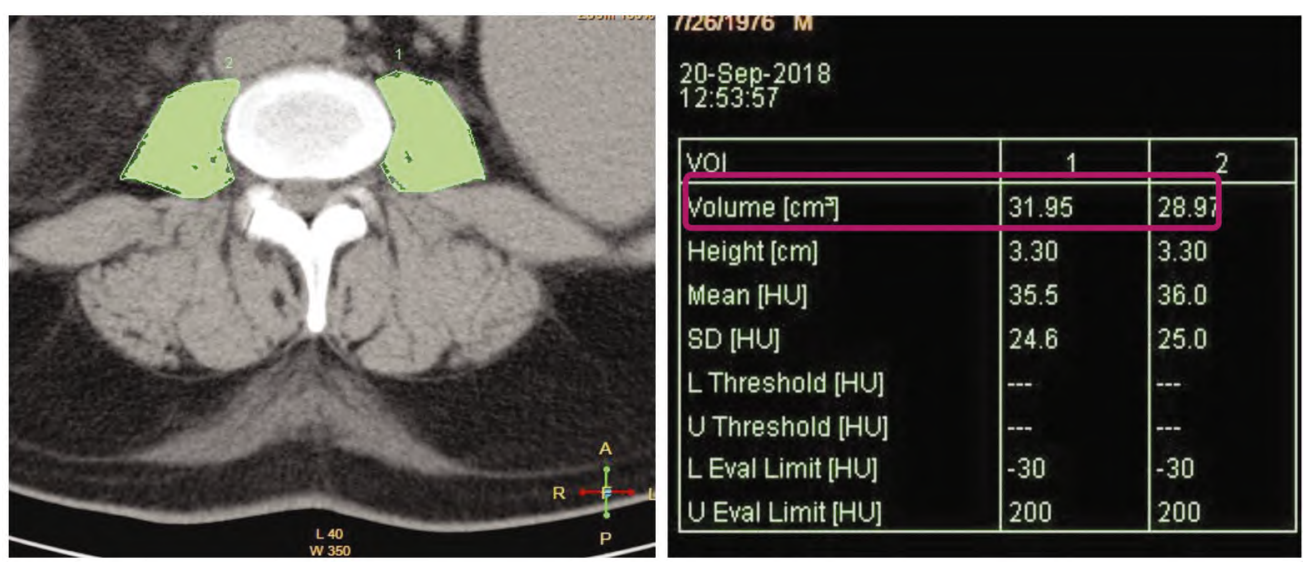

Fig. (1): CT psoas muscle volume which

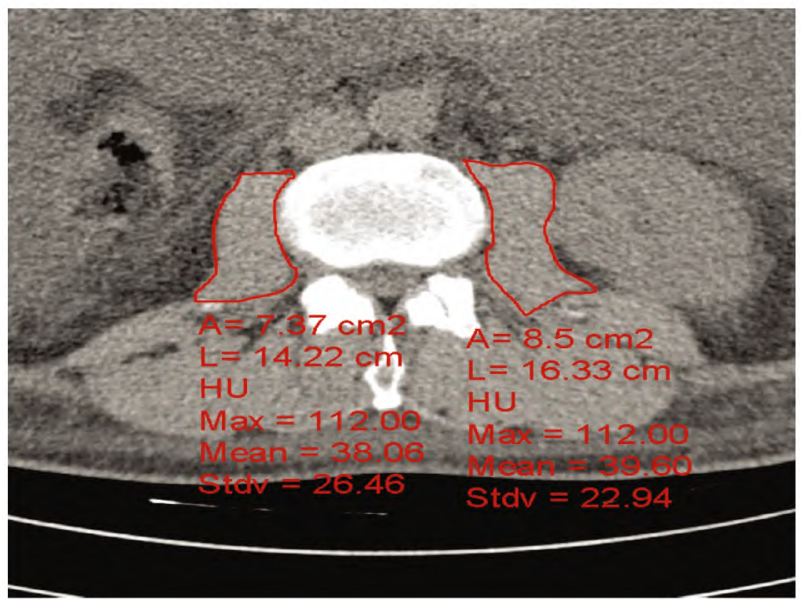

Fig. (2): CT psoas muscle cross sectional area.

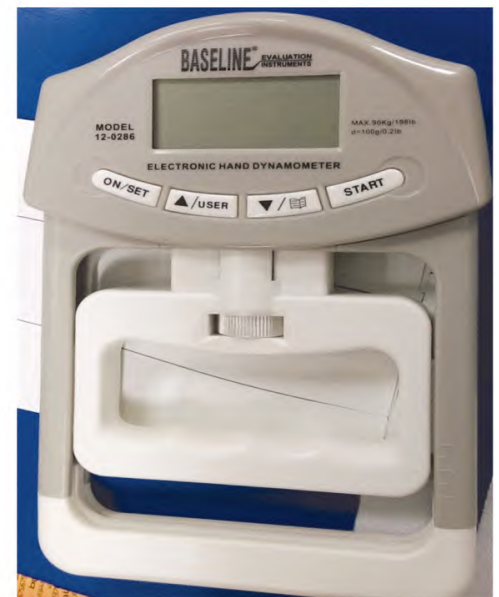

Fig. (3): Hand grip dynamometry. 

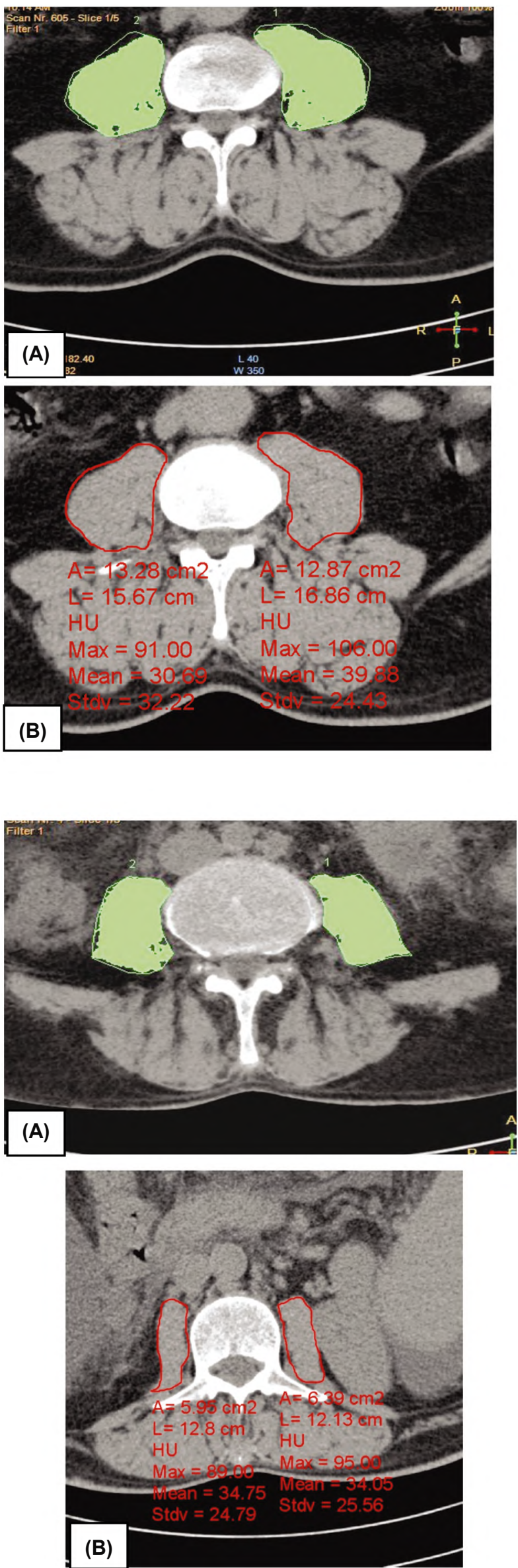

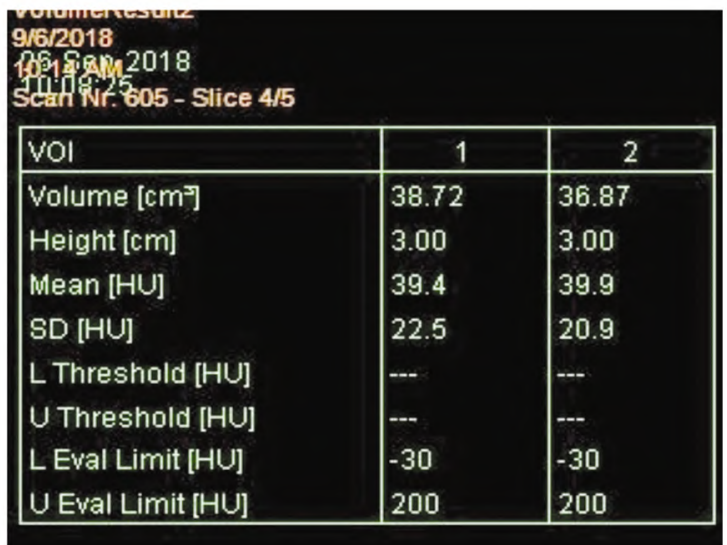

Fig. (4): (A) Psoas muscle volume and (B) Cross sectional area in healthy control subject.

BMI 23.6 \& HGS: 35.

Positive correlation with HGS.

\begin{tabular}{|c|c|c|}
\hline \multicolumn{3}{|l|}{ 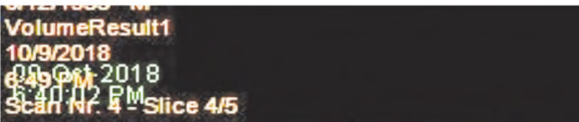 } \\
\hline Vol & 1 & 2 \\
\hline Volume $\left[\mathrm{cm}^{-}\right]$ & 30.80 & 28.77 \\
\hline Height $[\mathrm{cm}]$ & 3.30 & 3.30 \\
\hline Mean [HU] & 34.9 & 33.3 \\
\hline SD [HU] & 21.1 & 22.1 \\
\hline LThreshold [HU] & - & $\ldots$ \\
\hline U Threshold [HU] & - & $\ldots$ \\
\hline L Eval Limit [HU] & -30 & -30 \\
\hline U Eval Limit [HU] & 200 & 200 \\
\hline
\end{tabular}

Fig. (5): (A) Psoas muscle volume and (B) Cross sectional area in child B sore.

BMI 19 \& HGS: 26.5.

Positive correlation with HGS. 

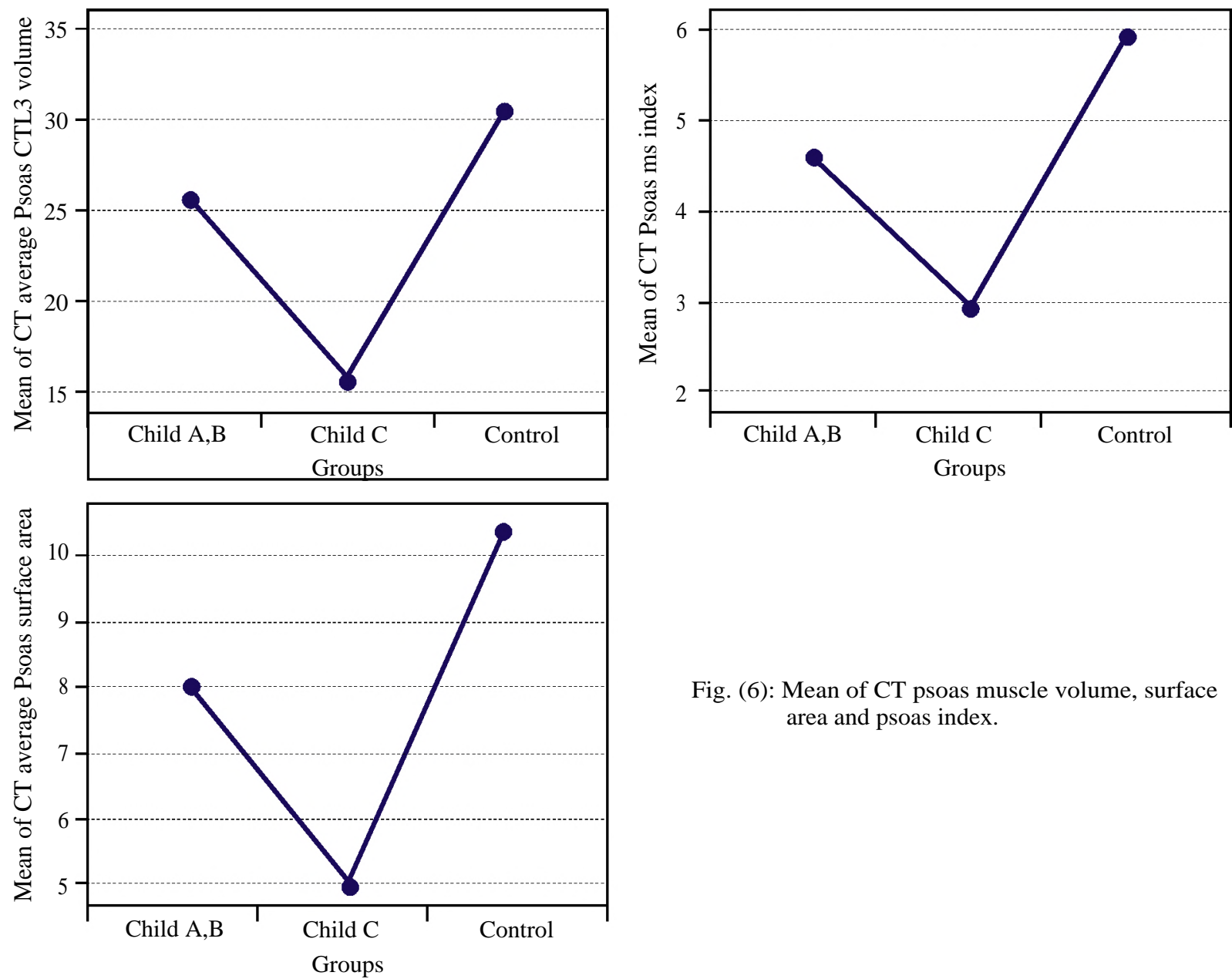

Fig. (6): Mean of CT psoas muscle volume, surface area and psoas index.

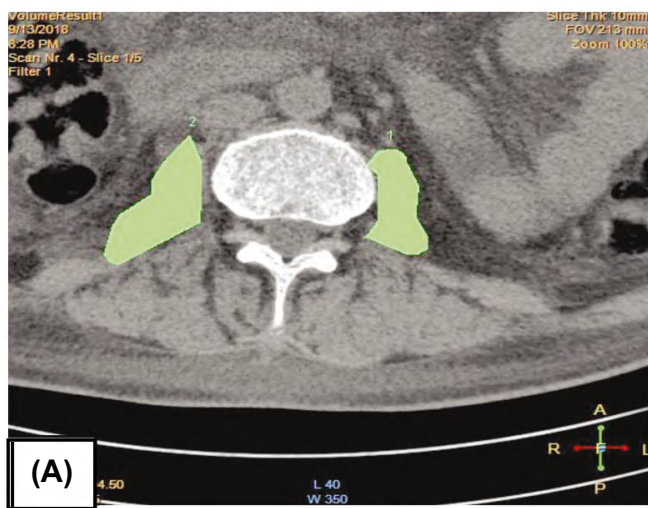

\begin{tabular}{|c|c|c|}
\hline $\mathrm{VOI}$ & 1 & 2 \\
\hline Volume $\left[\mathrm{cm}^{-}\right]$ & 12.93 & 18.17 \\
\hline Height $[\mathrm{cm}]$ & 2.70 & 2.70 \\
\hline Mean [HU] & 28.4 & 16.9 \\
\hline SD [HU] & 34.7 & 34.3 \\
\hline L Threshold [HU] & |..- & ... \\
\hline U Threshold [HU] & |-- & |-- \\
\hline LEval Limit [HU] & -1000 & -1000 \\
\hline U Eval Limit [HU] & 400 & 400 \\
\hline
\end{tabular}

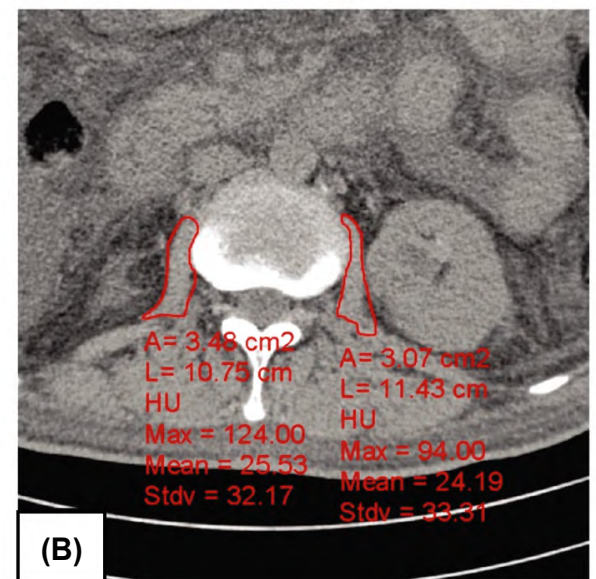

Fig. (7): (A) Psoas muscle volume and (B) Cross sectional area in child $\mathrm{C}$ sore.

BMI 17.4 \& HGS: 14.2

Positive correlation with HGS. 


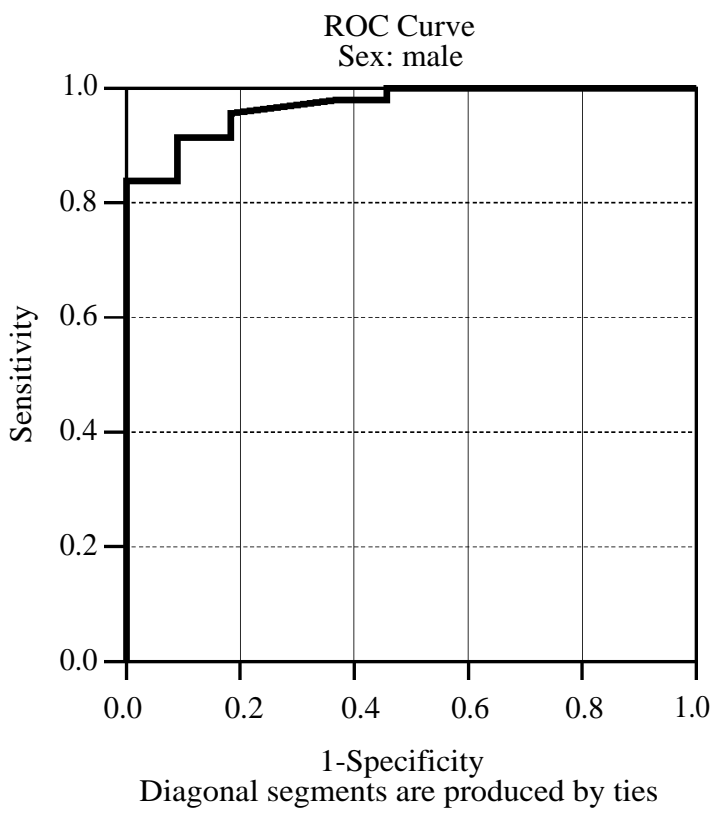

Fig. (8): Cut off value of male Psoas muscle index.

\section{Discussion}

Sarcopenia is characterized by progressive decline of muscle mass and strength, as well as functional capacity, having progressive relationship with malnutrition, chronic disease and malignancy [17]. In general muscle mass in sarcopnia is defined as being below two standard deviation of the normal healthy adult [18]

Severe muscle wasting or sarcopenia is one of the most common and frequently hidden complications in patients with cirrhosis, which negatively impact survival, quality of life, and response to stressor, such as infections and surgeries [19] Patients with malnutrition and/or sarcopenia have longer hospital stay, and increased in-hospital mortality [20].

Nowadays, calculating muscle mass with CT images acquired at level of L3, is an objective and reproducible method for assessing the degree of malnutrition and sarcopenia [18].

In our study, both CT parameters of the psoas muscle (Psoas Volume, surface area, and psoas muscle index) showed significant decrease in patients in comparison to control even in Child A patients.

Psoas muscle volume, surface area and psoas index were negatively correlated with (Child\& MELD) scores, and duration of hospital stay, the lower psoas muscle volume, surface area, psoas index the higher Child score, MELD score, duration of hospital stay with $(p<0.001)$.

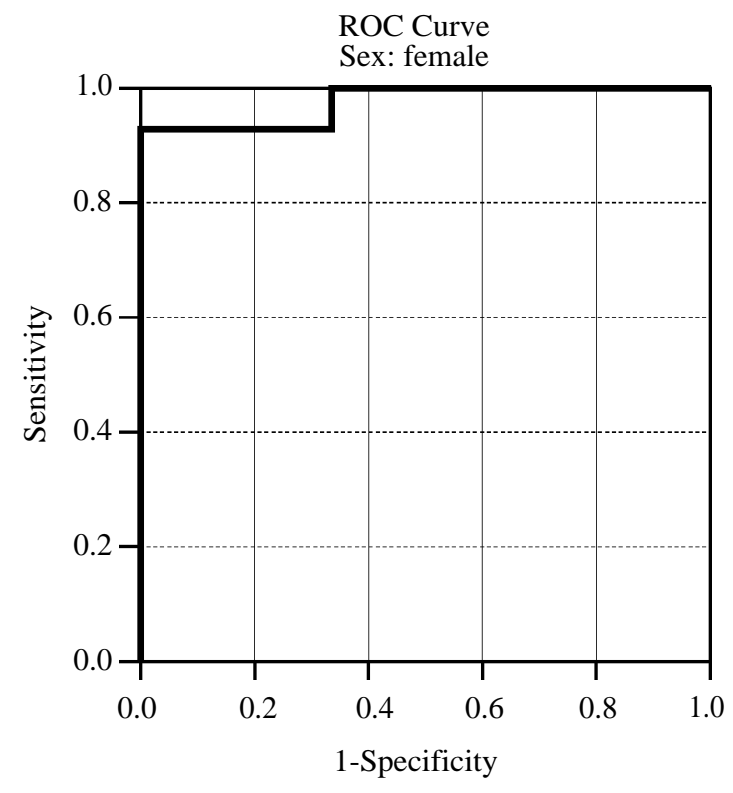

Fig. (9): Cut off value of female Psoas muscle index.

Kang SH, et al., [21] conducted Cohort study which enrolled 452 patients with cirrhosis (Child A (n: 215) Child B (n: 200) Child C (n: 37). L3 SMI was measured on CT imaging. Among the patients, 42\% (190/452) had sarcopenia [Child A (n: 93) B (n: 87) C (n: 10)], these results agree with our study, since sarcopenia started early even in Child A. Nearly 50\% (48.9\%) of patients who were either compensated or with early decompensated cirrhosis as in Child B patients were sarcopenic.

Gajula U and Murugan N, [22] performed a study which included 95 patients with liver cirrhosis. Similar to our study, they utilized CT examination at the level of L3, to estimate PMI (psoas muscle index). At their study Sarcopenia diagnosed by CT scan was correlated with (Child, MELD) scores and HGS. Gender specific Psoas muscle index cut-off values to define sarcopenia were derived from a pilot study of sixty healthy adults between 20-30 yrs whose values were $<5.7 \mathrm{~cm}^{2} / \mathrm{m}^{2}$ in males and $<3.5 \mathrm{~cm}^{2} / \mathrm{m}^{2}$ in females. Prevalence of sarcopenia assessed by PMI in their study was was found to be positively proportionate with the severity of chronic liver disease assessed by Child score $(p$-value $<0.05)$ and with MELD score $(p$ value $<0.005)$. Also sarcopenia was increased with decreased HGS ( $p$-value $<0.005$ ) and this agree with the results we reached in our study.

Ebadi M, et al., [23] established cut-off for PMI to predict mortality for (males $<5.1 \mathrm{~cm} / \mathrm{m}^{2}$; females $<4.3 \mathrm{~cm}^{2} / \mathrm{m}^{2}$ ), in our study we established Cut off value of PMI $\left(<4.66 \mathrm{~cm}^{2} / \mathrm{m}^{2}\right.$ for males and $<3.19$ 
$\mathrm{cm}^{2} / \mathrm{m}^{2}$ for female) using ROC curve of hand grip with high specificity $(100 \%)$ and sensitivity $(84 \%$ $\& 93 \%$ ), with (area under the receiver operating characteristic curve AUC 0.9) is similar to cut off values previously estimated by Ebadi et al., in 2018 in males but lower value for females.

HGS is a noninvasive, simple and quick method that can be applied in clinical and epidemiological studies [24].

Álvares-da-Silva MR and Silveira TR, [25] suggested that in early stages of cirrhosis, muscle strength measured by HGS should be used to evaluate for malnutrition and sarcopenia. In their study, among patients with cirrhosis, $88 \%$ were ChildPugh A and only $12 \%$ were Child-Pugh B, these patients were tested by handgrip strength (HGS) in the outpatient clinic $(n=50)$ and followed for one year to verify the incidence of major complications, the need for transplantation, and death. Among these patients, the prevalence of malnutrition was $63 \%$ by HGS $(p<0.05)$ and this agrees with our study, as we found that Hand grip showed significant positive correlation with anthropometric measurements (BMI, MAUC), and significant negative correlation with disease severity score (Child score) and with mortality risk (MELD) with ( $p$-value $>0.001)$.

Contrary to our results, a study conducted by Fernandes SA, et al., [26] showed no correlation between HGS and Child classification. They included patients who were mostly with compensated liver cirrhosis (91 of 129 patients were Child A, 27 were Child B, and only 9 were Child C). They tested the non-dominant hand and showed that HGS did not significantly decreased with increased severity of liver cirrhosis with $(p=0510)$.

The discrepancy between the results of our study and 26 may have originated from the fact that HGS was tested in the dominant hand in our study, while Fernandes et al., examined the nondominant hand.

In our study, HGS was positively correlated with CT L3 psoas volume, surface area and psoas muscle index with significant ( $p$-value $>0.001$ ).

Michela G, et al. [27] conducted a study on fifty-nine patients listed for LT aimed to verify the association between muscle wasting, determined by CT scan and HGS. HG dynamometer failed to correlate with CT (SMI) muscle evaluation in men ( $p$-value 0.73) and women ( $p$-value 0.69). The decrease in SMI was not significantly associated with a worse liver function (MELD vs. SMI, $p=0.33$; Child-Pugh vs. SMI, $p=0.76$ ) and this disagree with our study.

\section{Limitations:}

1- The most patients admitted in our hospital who have complications like hematemesis, hepatic coma, and others due to hepatic decompensation are of Child $\mathrm{C}$ group so patients of Child $\mathrm{A}$ and $\mathrm{B}$ patients are less in number. And most of Child C group patients have critical case so we stabilized the patients first.

2- Overflow of patients in all specialties in our hospital makes using CT scan for limited number of hepatic patients not all unlike other institutes which are specialized in hepatology.

3- There are no normal values for muscle thickness among Egyptians (normal general population) whether by ultrasound or CT measured.

4- We didn't follow-up patients after discharge for quality of life, readmissions and post hospital mortality.

\section{Strengths:}

1- We used CT (volume, surface area) to assess muscle thickness also we used HGS to asses muscle function.

2- To our best knowledge this is the first study using HGS to set cutoff value for the psoas index by CT.

\section{Conclusion:}

Testing for sarcopenia is very important in patients with chronic liver disease and cirrhosis, as sarcopenia is one of the important prognostic factors for the disease severity and poor outcome. $\mathrm{CT}$ is considered a gold standard cross sectional modality to test for sarcopenia, through measuring Psoas muscle volume, cross sectional area and PMI through single axial cut at the level of L3.

Also HGS is a simple bedside technique used for assessment of muscle function and sarccopenia, correlates positively with the measured CT parameters, as well as with the different scoring and grading systems for the severity of liver disease.

\section{References}

1- DAE HOE G., MOON K., YEON S., et al.: Clinical usefulness of psoas muscle thickness for the diagnosis of sarcopenia in patients with liver cirrhosis. Clinial and Milecular Hepatology, 24: 319-330, 2018.

2- DASARATHY S. and MERLI M.: Sarcopenia from mechanism to diagnosis and treatment in liver disease. $\mathrm{J}$. Hepatol., 65: 1232-1244, 2016.

3- KIM G., KANG S.H., KIM M.Y. and BAIK S.K.: Prognostic value of sarcopenia in patients with liver cirrhosis: 
A systematic review and meta-analysis. PLoS ONE, 12 (10): e0186990, 2017.

4- LUCIDI C., LATTANZI B., Di GREGORIO V., et al.: A low muscle mass in creases mortality in compensated cirrhotic patients with sepsis. Liver Int., 38 (5): 851-857, 2018.

5- JOHNSON T.M., OVERGARD E.B., COHEN A.E., et al.: Nutrition assessment and management in advanced liver disease. Nutr. Clin. Pract, 28: 15-29, 2013.

6- O'BRIEN A. and WILLIAMS R.: Nutrition in end-stage liver disease: Principles and practice. Gastroenterology, 134: 1729-1740, 2008.

7- MONTANO-LOZA A.J., DUARTE-ROJO A., MEZAJUNCO J., et al.: Inclusion of Sarcopenia With in MELD (MELD-Sarcopenia) and the Prediction of Mortality in Patients With Cirrhosis. Clin. Transl. Gastroenterol., 6: e102, 2015.

8- SINCLAIR M., GOW P.J., GROSSMANN M. and ANGUS P.W.: Review article: Sarcopenia in cirrhosis aetiology, implications and potential therapeutic interventions. Aliment Pharmacol. Ther., 43 (7): 765-777, 2016.

9- DUARTE-ROJO A., RUIZ-MARGA'IN A., MONTANOÑLoza A.J., MACíAS-RODRíGUEZ R.U., FERRANDO A., KIM W.R.: Exercise and physical activity for patients with end-stage liver disease: Improving functional status and sarcopenia while on the transplant waiting list. Liver Transplant, 24 (1): 122-139, 2018.

10- Carey E.J., Lai J.C., Wang C.W., et al.: "A multicenter study to define sarcopenia in patients with end-stage liver disease", Liver Transplantation, Vol. 23, No. 5, pp. 625633, 2017.

11-GIUSTO M., LATTANZI B., ALBANESE C., GALTIERI A., FARCOMENI A., GIANNELLI V., et al.: Sarcopenia in liver cirrhosis: The role of computed tomography scan for the assessment of muscle mass compared with dualenergy X-ray absorptiometry and anthropometry. Eur. J. Gastroenterol. Hepatol., 27: 328-334, 2015.

12- FLOOD A., CHUNG A., PARKER H., et al.: The use of hand grip strength as a predictor of nutrition status in hospital patients. Clin. Nutr., 33: 106-14, 2014.

13- RANTANEN T., VOLPATO S., FERRUCCI L., et al.: Handgrip strength and cause-specific a total mortality in older disabled women: Exploring the mechanism. J. Am. Geriatr Soc., 51: 636-641, 2003.

14- NORMAN K., STOBÄUS N., GONZALEZ M.C., SCHULZKE J.D. and PIRLICH M.: Hand grip strength: Outcome predictor and marker of nutritional status. Clin. Nutr., Apr. 30 (2): 135-42. doi: 10.1016/j.clnu.2010.09.010. Epub 2010 Oct 30. PMID: 21035927, 2011.

15- McLEAN R.R., SHARDELL M.D., ALLEY D.E., et al.: Criteria for clinically relevant weakness and low lean mass and their longitudinal association with incident mobility impairment and mortality: The Foundation for the National Institutes of Health (FNIH) Sarcopenia
Project. J. Gerontol. A Biol. Sci. Med. Sci., 69: 576-583, 2014.

16- RONG S., WANG L., PENG Z., et al.: The mechanisms and treatments forsarcopenia: Could exosomes be a perspective research strategy in the future? J. Cachexia Sarcopenia Muscle, 11: 348-365, 2020.

17- CRUZ-JENTOFT A.J., BAEYENS J.P., BAUER J.M., et al.: European Working group on sarcopenia in older people. Sarcopenia: European consensus on definition and diagnosis: Report of the European Working Group on Sarcopenia in Older People. Age Ageing, 39 (4): 41223. (doi: 10.1093/ageing/afq034), 2010.

18- ERKAN M., AHMETOGLU A., CANSU A. and ERKUT M.: Evaluation of Sarcopenia and Investigation of Prognostic Value Of Sarcopenia Using Psoas Muscle Area on Computed Tomography in Patients with Liver Cirrhosis. European Journal of Medical and Educational Technologies, 14 (3): em2111. https://doi.org/ 10.30935/ejmets /11094, 2021.

19- MONTANO-LOZA A.J., MEZA-JUNCO J., BARACOS V., et al.: Severe muscle depletion predicts postoperative length of stay but is not associated with survival after liver transplantation. Liver Transplant, 20: 640-648, 2014.

20- SAM J. and NGUYEN G.C.: Protein-calorie malnutrition as a prognostic indicator of mortality among patients hospitalized with cirrhosis and portal hypertension. Liver Int: Official journal of the International Association for the Study of the Liver, 29: 1396-1402, 2009.

21- KANG S.H., JEONG W.K., BAIK S.K., et al.: Impact of sarcopenia on prognostic value of cirrhosis: Going beyond the hepatic venous pressure gradient and MELD score Journal of Cachexia, Sarcopenia and Muscle, 9: 860-870, 2018.

22- GAJULA U. and MURUGAN N.: Assessment of sarcopenia in patients with chronic liver disease, Volume 8 , Supplement 1, Page S6, 2018.

23- EBADI M., WANG C.W., LAI J.C., et al.: Poor performance of psoas muscle index for identification of patients with higher waitlist mortality risk in cirrhosis. J. Cachexia Sarcopenia Muscle, 2018.

24- PLAUTH M., CABRE E., RIGGIO O., et al.: ESP enteral nutrition guidelines on enteral nutrition: liver disease. Clin. Nutr., 25: 285-294, 2006.

25- ÁLVARES-da-SIVA M.R. and SILVEIRA T.R.: Handgrip strength or muscle mass in cirrhotic patients: Who is the best? Nutrition, 22: 218-219, 2006.

26- FERNANDES S.A., de MATTOS A.A., TOVO C.V., et al.: "Nutritional evaluation in cirrhosis: emphasis on the phase angle," World Journal of Hepatology, Vol. 8, No. 29: pp. 1205-1211, 2016.

27- MICHELA G., BARBARA L., CARLINA A., et al.: European journal of gastroenterology and hepatology, 27 (3) DOI: 10. 1097/MEG. 0000000000000274, 2015. 


\section{تقييم العلاقة بين شدة الساركوبينيا

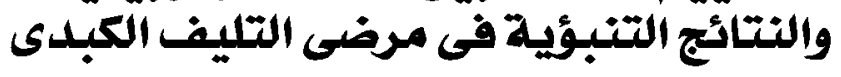

عرُف الفريق العامل الأقوبى معنى الساركوبينيا لدى كبار السن بأنه وجود كل من كلة الوضلات المنخفضة ووظائف العضلات المنخفضة (القوة أى الأداء).

تعتبر الساركويينيا سمة شائعة لسوء التغذية لدى المرضى الذين يعانون من تليف الكبد ومن المعترف به نطاق واسع أنه مؤثشر مستقل

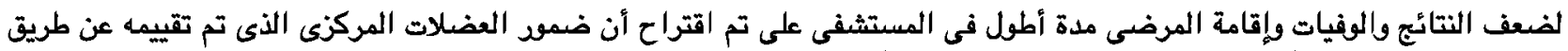

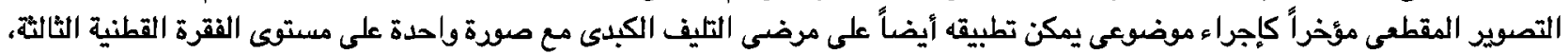
وهو ممثل صالح لكتلة عضلات الجسم بـوالكملها.

والهدف من هذه الدراسدة: تقييم شدة الساركوبينيا فى مرضى تلف الكبد وعلاقته بالنتائج التشخيصية التقليدية لتليف الكبد، مثل تقييم

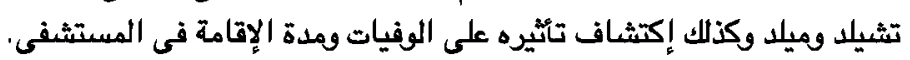

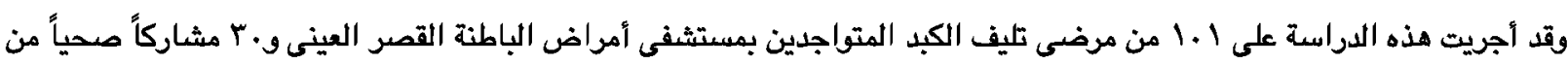

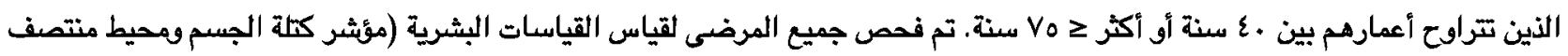

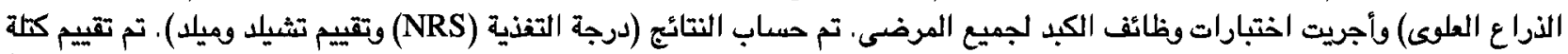

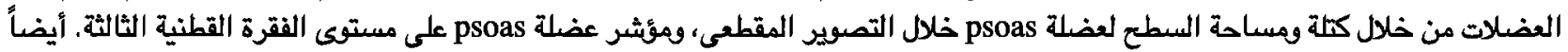

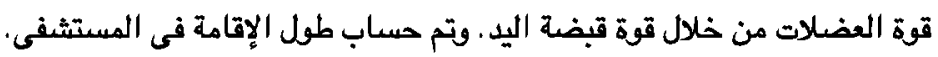

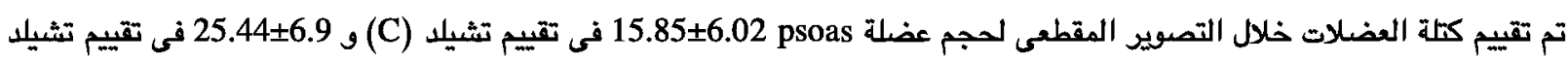

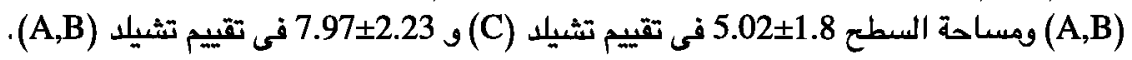

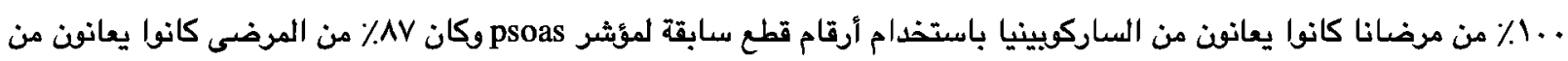

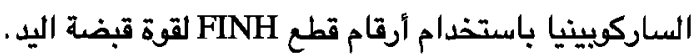

وتم عمل أرقام قطع لقيمة مؤثر عضلة psoas باستخدام FINH لقوة قبضة اليد وكانت (7 .ع سم/م Y) للذكود و 19 .r للإناث). وقد وجد علاقة سلبية بين كثلة ومساحة السطح عضلة psoas مع تقييم تشيلد وميلد ودرجة التفذية (NRS) ومع مدة الإقامة فى المستشفى مئى

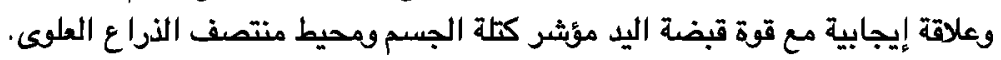

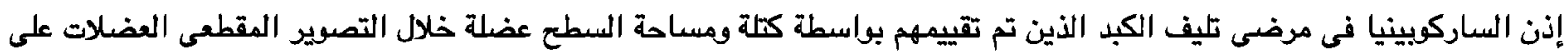

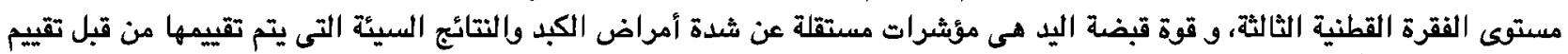

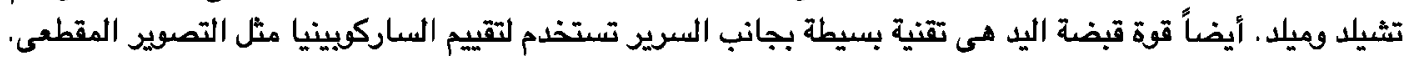

